\title{
Euclidean Feature Set Based on Modified Characteristic Polarization States
}

\author{
Faisal Aldhubaib
}

Electronics Department, College of Technological Studies, Public Authority of Applied Education, Kuwait

\begin{abstract}
This paper presents a feature set selection procedure, which is implemented in the frequency domain and involves developing a modified CPS feature set that incorporates power information. Metric distances between the respective modified CPS at several target resonant frequencies are computed and are used to form the final feature set. Stationary individual targets in free space were used to display the theoretical feasibility of this feature selection algorithm.
\end{abstract}

Keywords: Characteristic polarization states, radar feature set, resonances theory, Singularity Expansion Method, Euclidean distance

\section{Introduction}

There are many techniques and algorithms used to recognize radar targets. Descriptions and details of their associated formulation, advantages and disadvantages may be found in, for example $[1,2]$. Target identification based on resonance excitation (e.g. the Extinction pulse technique of [3-5]), uses baseband pulse excitation to reveal the target natural resonance modes. The mode resonant frequencies are target dependent, have enhanced returns and independent of aspect; thus making them a prime candidate for inclusion in any target feature set description. The resonance frequencies may be estimated directly from the frequency domain by locating the peaks in the broad frequency response; or otherwise, extracted or estimated from the target late-time portion of the temporal response by matrix-pencil-of-function (MPOF) method[6].

Nevertheless, while a resonance feature set contains information about the global dimensions of the target, it cannot reflect the target shape attributes such as elongation, symmetry and tilt; unless a polarization descriptor is incorporated[7, 8]. In the polarization domain, the target functions as a polarization transformer of the incident wave polarization state to a scattered wave polarization state. The polarization state of the wave or antenna can generally be described by the Stokes vector for a power radar return (i.e. incoherent case). Thus considered, the polarization state can also be represented as a point on the Poincaré sphere, where the rectangular coordinates of the point correspond to three of the components of the Stokes vector. Target polarization scattering is modelled mathematically by Kennaugh power matrix for the general incoherent returns[9]. A polarization scattering matrix should be able to unfold the symmetry and the tilt of the target, the number of bounces of the reflected wave and its ability to polarize incident unpolarized radiation, i.e. elongation [10]. In addition, the Kennaugh matrix can also be used to optimize the received power (using a Lagrange multiplier method) as a function of the antenna polarization state, where the CPS represents critical points (maxima, minima, and null) in the co-polarized (co-pol) and cross-polarized (x-pol) power spectra. Therefore, it is possible to represent the target polarization characteristics by its CPS set, which in turn form the famous polarization (or Huynen) fork on the Poincare sphere. In addition to the optimum co-pol and x-pol powers, the relationship between the CPS set (has polarization fork shape) is invariant to the antenna tilt or polarization basis.

In this work, a set of target resonant returns is used as a way to first classify targets and then to incorporate their CPS as a way of discriminating individual targets within the same class. The Lagrange multiplier method is used to optimize the power received in both co-pol and x-pol channels and to subsequently derive the ten CPS. Pre-selected CPS are then modified by their respective co-pol and x-pol powers to form a modified set of these CPS, and then relationships (Euclidean distances) between these various CPS are used as the final feature set. However, a difficulty remains in considering the power optimisation algorithm when the return is partially polarized with some degree of spectral spread due to noise, clutter, or even refraction in the transmission medium. Nonetheless, if the spectral spread is small compared to the resonance spectral spread of interest, the received return can be considered quasi-monochromatic, and therefore, can be handled by signal averaging achieved by means of pulse integration[11].

This paper is organized as follows: Section 2 gives the needed formulas of the polarization scattering and the optimization process used to arrive at the characteristic polarization states. Section 3 presents the algorithm used to modify the states according to associated powers and shows how the feature set is derived quantitatively from the original CPS data. Section 4 presents the feature set sensitivity to changes in the target shape via example of two geometrically very similar targets Section 5 reaches conclusions and indicates where further work is required.

\section{Formulas}

\subsection{Scattering Matrix}

The Stokes vector is used to describe the polarization state of a wave or antenna for completely (and even partially) polarized waves as follows

$$
\mathbf{g}=\left[g_{0} g_{1} g_{2} g_{3}\right]^{\mathrm{T}}
$$

Subject to: $g_{0}=\left(g_{1}{ }^{2}+g_{2}{ }^{2}+g_{3}{ }^{2}\right)^{1 / 2}$ for fully polarized wave. denotes the transpose. The $g_{o}$ denotes the wave intensity or total instantaneous power, $\mathrm{g}_{1}$ gives the portion of the wave that is horizontally or vertically polarized, $\mathrm{g}_{2}$ gives the portion 


\section{International Journal of Science and Research (IJSR) \\ ISSN (Online): 2319-7064}

Index Copernicus Value (2013): 6.14 | Impact Factor (2015): 6.391

of the wave that is linearly oriented at $\pm 45^{\circ}$ and $g_{3}$ gives the portion of the wave that is left or right circularly polarized, respectively. In the case of a partially polarized wave due to clutter or noise, ensemble-time averaging of Stokes vector is used. In general, the second moment properties (i.e. power terms) of a scattering wave at a single frequency be represented by the Kennaugh formulism, as follows

$$
[\mathrm{K}]=\mathrm{R}\left(\left|[\mathrm{S}] \otimes[\mathrm{S}]^{*}\right|\right) \mathbf{R}^{-1}, \quad \mathbf{R}^{-1}=\frac{1}{2} \mathbf{R}^{{ }^{\mathrm{T}}}
$$

Here

$$
\mathbf{R}=\left(\begin{array}{cccc}
1 & 0 & 0 & 1 \\
1 & 0 & 0 & -1 \\
0 & 1 & 1 & 0 \\
0 & j & -j & 0
\end{array}\right)
$$

For an orthogonal linear polarization basis, e.g. $(h, v)$, the polarization scattering matrix (PSM) $\mathbf{S}$ is expressed as follows

$$
\mathbf{S}=\left[\begin{array}{ll}
S_{k k} & S_{k v} \\
S_{v k} & S_{v v}
\end{array}\right]
$$

The subscripts denote the transmitter and receiver polarization directions, where $h h$ and $v v$ denote the co-polarized scattering directions or channels, while $h v$ and $v h$ denote the cross-polarized scattering channels (reciprocal for monostatic case). The product $\left(\left|[\mathbf{S}] \otimes[\mathbf{S}]^{*}\right|\right)$ gives the entire correlation relations between the $\mathbf{S}$ matrix elements, and is defined as the Kronecker product of the PSM and its conjugate. The Kennaugh matrix $\mathbf{K}$ relates incident and scattered Stokes vectors $\mathbf{g}_{\mathbf{i}}$ and $\mathbf{g}_{\mathrm{s}}$ as follows

$$
g_{s}=K \cdot g_{i}
$$

\subsection{Received Power}

For a monostatic antenna case, the power at the receiver terminals can be expressed in terms of antenna Stokes vector $\mathbf{g}_{\mathrm{a}}$. and power matrix $\mathbf{K}$ as

$$
P_{\mathrm{sec}}=\mathrm{g}_{\mathbf{2}} \mathbf{K g}_{\mathrm{z}}^{\mathrm{T}}
$$

On reception, the wave is split into co-pol and x-pol channels with the power at the antenna terminals established separately for each channel. Thus the Kennaugh matrix requires a factorization into two new Kennaugh matrices to accommodate both the co-pol and x-pol configurations. The associated received power in the co-pol channel and x-pol channel can be written as

$$
\begin{aligned}
& P_{\mathrm{c}}=\mathbf{g}_{\mathbf{a}} \mathbf{K}_{\mathrm{c}} \mathbf{g}_{\mathrm{a}}^{\mathrm{T}} \\
& P_{\mathbf{x}}=\mathbf{g}_{\mathbf{a}} \mathbf{K}_{\mathbf{x}} \mathbf{g}_{\mathbf{a}}^{\mathrm{T}}
\end{aligned}
$$

Where, in general

$$
\mathbf{K}_{\mathrm{c}}=\frac{1}{2}\left(\begin{array}{cccc}
1 & 0 & 0 & 0 \\
0 & 1 & 0 & 0 \\
0 & 0 & 1 & 0 \\
0 & 0 & 0 & -1
\end{array}\right) . \mathbf{K} \text { and } \mathbf{K}_{\mathrm{x}}=\frac{1}{2}\left(\begin{array}{cccc}
1 & 0 & 0 & 0 \\
0 & -1 & 0 & 0 \\
0 & 0 & -1 & 0 \\
0 & 0 & 0 & 1
\end{array}\right) . \mathbf{K}
$$

Here the subscript , ${ }^{c e}$ and , $\mathrm{X}^{\text {ee }}$ denotes the co-pol and the orthogonal x-pol configurations, respectively. Both equations (6) and (7) are essential for deriving the CPS and their associated power levels using the Lagrangian multiplier method, where the optimum ga that gives the optimum co- and $\mathrm{x}$-powers is seeked.

\subsection{Characteristic Polarization States}

The Kennaugh power optimization will be carried out separately for the co-pol and x-pol channels, where the received powers will be maximized and minimized by applying the Lagrangian multiplier method to the antenna Stoke vector $\mathbf{g}_{\mathrm{a}}$ with the constraint that $g_{\mathrm{o}}$ is unity. The constraint condition $\Phi$, written in terms of the $\mathbf{g}_{\mathbf{a}}$ variables is then defined as

$$
\Phi=\sqrt{g_{1}^{2}+g_{2}^{2}+g_{3}^{2}}-1=0
$$

The variations of the $\mathbf{g}_{\mathrm{a}}$ variables will lead to maximizing or minimizing the received power at the receiver terminals, where the optimum co-pol and x-pol powers can be found by simultaneously solving for the first partial derivatives of $P_{c}$ and $P_{x}$ in terms of the stokes variables. This procedure results in three simultaneous partial derivative equations for each coand $\mathrm{x}$ - polar power, as follows:

$$
\begin{array}{ll}
\frac{\partial P_{c}}{\partial g_{n}}-\mu \frac{\partial \Phi}{\partial g_{n}}=0, & n=1,2,3 . \\
\frac{\partial P_{z}}{\partial g_{n}}-\mu \frac{\partial \Phi}{\partial g_{n}}=0, & n=1,2,3 .
\end{array}
$$

Where $\mu$ is the Lagrangian multiplier and gives the rate of change of the power quantity being optimized as a function of the constraint variables. Solving (9) gives two pair of co-pol type CPS, they are the orthogonal co-pol max pair $\left(\mathrm{cm}_{1}, \mathrm{~cm}_{2}\right)$ represented by the conjugate Stokes vector pair $\mathbf{g}\left(\mathrm{cm}_{1,2}\right)$ and the co-pol null pair $\left(\mathrm{cn}_{1}, \mathrm{cn}_{2}\right)$ represented by the Stokes vector pair $\mathbf{g}\left(\mathrm{cn}_{1,2}\right)$. The latter are not orthogonal but define a polarization characteristic angle. Whereas, solving (10) gives three pairs of $x$-pol type CPS, they are the orthogonal x-pol max pair $\left(\mathrm{xm}_{1,2}\right)$ represented by the conjugate Stokes vector pair $\mathbf{g}\left(\mathrm{xm}_{1,2}\right)$, the orthogonal $\mathrm{x}$-pol saddle pair $\left(\mathrm{xs}_{1,2}\right)$ represented by the conjugate Stokes vector pair $\mathrm{g}\left(\mathrm{xs}_{1,2}\right)$, and the orthogonal $\mathrm{x}$-pol null pair $\left(\mathrm{xn}_{1,2}\right)$ represented by the conjugate Stokes vector pair $\mathbf{g}\left(\mathrm{xn}_{1,2}\right)$.

The CPS set represents the critical points in the co-pol and $\mathrm{x}$-pol power spectra, but without any information about the level of power at these points. Such power levels associated with a CPS set can be found by inserting the derived CPS back into (6) and (7), then calculating their associated co-pol and $\mathrm{x}$-pol powers. 


\section{International Journal of Science and Research (IJSR) \\ ISSN (Online): 2319-7064}

Index Copernicus Value (2013): 6.14 | Impact Factor (2015): 6.391

The CPS feature has the following physical attributes: firstly, the target characteristic angle is a measure the target elongation degree; secondly, the symmetry information is reflected in the ellipticity angle of the maximum $\mathrm{cm}$ state $\left(0^{\circ}\right.$ for symmetrical target, \pm 45 for totally nonsymmetrical target); thirdly, the $\mathrm{g}_{1}, \mathrm{~g}_{2}$ elements of $\mathbf{g}\left(\mathrm{cm}_{1}\right)$ determines the tilt degree of the target major axis.

Until now, the CPS features are restricted to be on the surface of a polarization sphere since all CPS are assumed to have unit length. However, the polarization space inside the sphere surface can embody the CPS by incorporating their associated optimal power instead of assuming they have unit powers. This idea of modifying the CPS according to their associated power will be the core motivation of the proposed identification algorithm presented next.

\section{Algorithm}

The algorithm implements a metric measure criterion to derive relationships between respective modified characteristic polarization states when the latter are evaluated at the target natural resonance frequencies. After derived, the unit vectors CPS are weighted by their associated power levels to create a new set of modified CPS. Finally, a distance measure criterion is used to represent the changes of each respective modified CPS as a function of the target natural resonances. These distances are then assigned as the final feature set. They are invariant to range, target tilt orientation along the antenna boresight direction and can be used to improve resonance based discrimination algorithms.

\subsection{Modified CPS}

Recalling that the Stokes variables $\left(g_{1}, g_{2}, g_{3}\right)$ give the position of the polarization state on the polarization sphere, while $g_{\text {o }}$ represent its Euclidean distance from the sphere origin or radius length. Therefore, tailored by their associated power, the modified CPS set will lie inside the unit sphere with different radius lengths according to their respective powers. Now it's beneficial to select only non-redundant CPS to derive the proposed feature set (i.e. array of Stokes vectors). For the current case of symmetrical target, this required removing one of the co-pol null states, one of the x-pol maximum states and both pairs of x-pol saddles and x-pol nulls states. As a result, the feature set included the Stokes pairs $\mathbf{g}\left(\mathrm{cm}_{1,2}\right), \mathbf{g}\left(\mathrm{cn}_{1}\right)$ and $\mathbf{g}\left(\mathrm{xm}_{1}\right)$, which will be modified by their respective power factors, as follows

$$
\begin{array}{r}
\mathbf{g}^{\mathrm{m}}\left(\mathrm{cm}_{1,2}\right)=P_{\mathrm{c}}\left(\mathrm{cm}_{1,2}\right) \times \mathbf{g}\left(\mathrm{cm}_{1,2}\right) \\
\mathbf{g}^{\mathrm{m}}\left(\mathrm{cn}_{1}\right)=P_{\mathbf{x}}\left(\mathrm{cn}_{1}\right) \times \mathbf{g}\left(\mathrm{cm}_{1}\right) \\
\mathbf{g}^{m \infty}\left(\mathrm{xm}_{1}\right)=P_{\mathbf{x}}\left(\mathrm{xm}_{1}\right) \times \mathbf{g}\left(\mathrm{xm}_{1}\right)
\end{array}
$$

The power factors were chosen intuitively for the following reasons. The power levels associated with the critical points $\left(\mathrm{cm}_{1,2}\right)$ and $\left(\mathrm{xm}_{1}\right)$ are $P_{\mathrm{c}}\left(\mathrm{cm}_{1,2}\right)$ and $P_{\mathrm{x}}\left(\mathrm{xm}_{1}\right)$, respectively. Whereas for the critical point $\left(\mathrm{cn}_{1}\right)$, the associated power level is zero in the co-pol spectrum for a symmetrical target and fully polarized wave, instead the $\left(\mathrm{cn}_{1}\right)$ associated power appears in the parameter $P_{\mathrm{x}}\left(\mathrm{cn}_{1}\right)$, and therefore is used.
Hence, the proposed modified CPS feature set $\mathbf{F}$ consist of four modified CPS and can be expressed as a matrix of four characteristic Stokes vectors as follows

$$
\mathbf{F}=\left[\mathbf{g}^{\mathrm{mo}}\left(\mathrm{cm}_{1}\right), \mathbf{g}^{\mathrm{mo}}\left(\mathrm{cm}_{2}\right), \mathbf{g}^{\mathrm{mo}}\left(\mathrm{cn}_{1}\right), \mathbf{g}^{\mathrm{mo}}\left(\mathrm{xm}_{1}\right)\right]
$$

\subsection{Distance Measure}

This modification can be then repeated at all resonant frequencies of interest. For different angles of aspect, the feature set will create a distribution of modified CPS positions within the feature space. This distribution will generate a series of training prototypes, which in turn can be catalogued and used in an identification procedure (not covered here). The Euclidean relationships between the respective modified CPS of the $N$ separate resonances are computed using a simple metric distance measure. These Euclidean distance quantities will be representative of the final radar polarization feature set.

Such Euclidean distance between two respective CPS at the $n^{\text {th }}$ resonance and the $m^{\text {th }}$ resonance is defined as

$$
d_{n, m}=\left(\begin{array}{c}
\left.\left(g_{1}^{n}-g_{1}^{m}\right)^{2}+\right)^{\frac{1}{2}} \\
\left(g_{2}^{n}-g_{2}^{m}\right)^{2}+ \\
\left(g_{3}^{n}-g_{3}^{m}\right)^{2}
\end{array}\right)^{2}
$$

where $n \neq m$ and $n, m \in[1, \ldots, N]$ are the respective resonance orders. Henceforth, $1 / 2(\mathrm{~N}-1) . \mathrm{N}$ metric distances will be computed for $N$ resonances. The distances are then arranged in the order $(n, m)=(1,2), \ldots,(1, N) ;(2,3), \ldots,(2, N) ; .$. , $(N-1, N)$. For $N=3$; three distances $\mathrm{d}_{1,2}, \mathrm{~d}_{1,3}$, and $\mathrm{d}_{2,3}$ for each Stokes column vector are computed, thus a total of twelve distances are computed for a single aspect angle.

\section{Results}

\subsection{Target Geometry and Natural Resonances}

To illustrate the robustness of this radar feature set developed, a two similar canonical shaped target of Perfect Electric Conducting (PEC) bodies of revolution (BOR) were considered and shown in Figure 1. The ellipsoid target is $24 \mathrm{~cm}$ long with $8 \mathrm{~cm}$ diameter, and the cylinder target is $18 \mathrm{~cm}$ long with $8 \mathrm{~cm}$ diameter. The cylinder will serve as similar sized clutter-object (i.e. a radar, ,confuser") to benchmark the discrimination ability of the proposed signature. When defining a target class, it is customary to use one or more of the target physical features to define the class, in this case, the target major (horizontal) and minor (vertical) circumferences lengths were used to define a certain class of targets. This can be validated by the similar resonating behavior of the two targets class for a normal incident plane wave and up to the second dominant resonant frequency, as depicted in Figure 2. 


\section{International Journal of Science and Research (IJSR) \\ ISSN (Online): 2319-7064}

Index Copernicus Value (2013): 6.14 | Impact Factor (2015): 6.391

In the current analysis and simulation stages, a Gaussian plane wave incidence on the shapes of Figure 1 was assumed, where the excitation frequency was linearly stepped from DC to $2.5 \mathrm{GHz}$ in dual orthogonal directions to generate the target baseband broad response; although in practice, the usage of narrow bandwidth time domain excitation could be substituted for the stepped frequency domain excitation. The fully polarized far field complex frequency responses of the backscatter in the co-pol (HH and VV) and x-pol (HV and $\mathrm{VH}$ ) channels were computed (magnitude and phase) using a Method of Moments (MoM) algorithm [31], then filtered by a Gaussian window. The resonant peaks in the magnitude spectra were used as an approximation to the natural resonance frequencies, and the polarization scattering matrix was determined for each such single resonance frequency. Once the scattering matrix was determined, the optimization algorithm was implemented at these frequencies to obtain the proposed radar feature set.

The two dominant resonances in the frequency responses are related to the major (horizontal) and minor (vertical) circumferences of the target and since these circumference lengths are similar for both targets, the two target frequency profiles are expected to have relatively similar resonant behavior up to $1.2 \mathrm{GHz}$. For target broadside (or normal) incidence, the fundamental resonance in the $\mathrm{HH}$ channel occurs at around $550 \mathrm{MHz}$, while the fundamental resonance in the VV channel occurs around $1150 \mathrm{MHz}$. Due to target symmetry about the incident planes ( $\mathrm{H}$ and $\mathrm{V}$ planes), the $\mathrm{x}$-pol channels ( $\mathrm{HV}$ and $\mathrm{VH}$ ) frequency responses were negligible and thus omitted. For a clearer view of the resonance peaks in the frequency profiles, oblique azimuth angle incidence is preferred where the first three resonances are clearly similar with slight shifts for the higher harmonics.

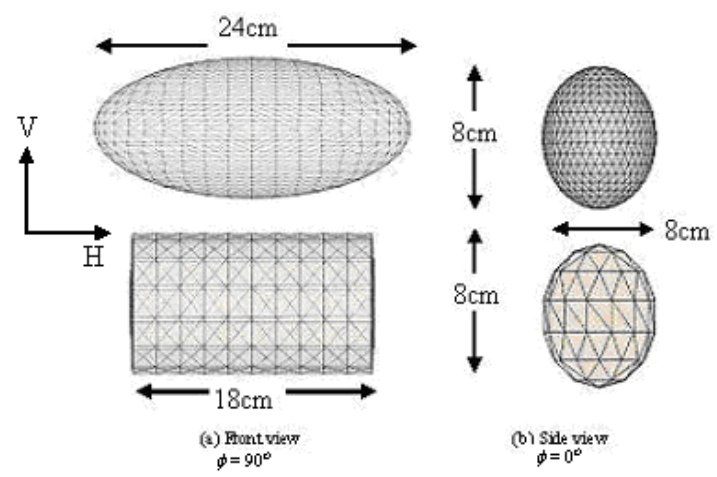

Figure 1: Geometry of the ellipsoid and cylinder.

\subsection{Modified Characteristic Polarization States}

The results for the modified characteristic polarization states of the two targets for normal incidence are shown in Table I. For tilt variation purposes, the modified characteristic polarization states of the ellipsoid with its major axis rotated $45^{\circ}$ about the $y$-axis is also included in the table. axis is in the horizontal direction.

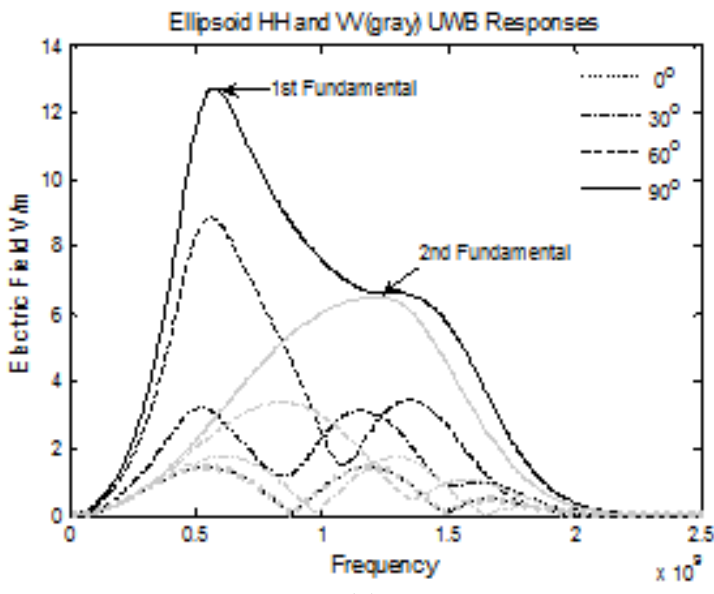

(a)

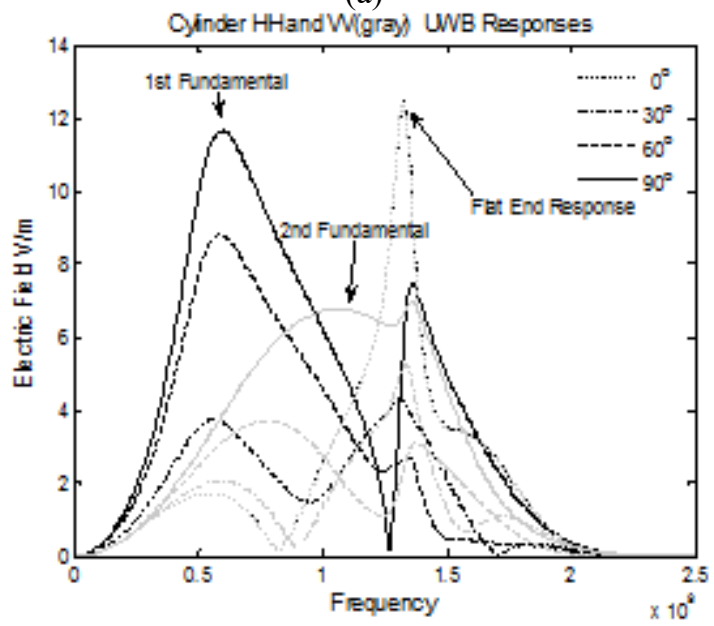

(b)

Figure 2: The HH (black) and VV (gray) channels UWB frequency responses as function of azimuth angles $(0,30,60,90)^{\circ}$ for (a) the Ellipsoid (b) the Cylinder.

Clearly from the optimization results for the ellipsoid its observed for azimuth of $90^{\circ}$, that the power level corresponding to $\mathrm{g}_{\mathrm{cm} 1}$ is always concentrated in the horizontal portion of the wave for the $1^{\text {st }}, 2^{\text {nd }}$ and $3^{\text {rd }}$ resonances; indicating that the target major. Additionally, the power level corresponding to $\mathrm{g}_{\mathrm{cm} 2}$ is almost absent for the $1^{\text {st }}$ resonance but reaches its maximum at the $2^{\text {nd }}$ resonance, indicating that the target minor axis is in the vertical direction. Finally, it is noted that the power ratio between $\mathrm{g}_{\mathrm{cm} 1}$ and $\mathrm{g}_{\mathrm{cm} 2}$ can indicate how linear the target is. All these observations are consistent with the horizontal and vertical frequency responses of Figure 2. On the other hand, the cylinder results for the $2^{\text {nd }}$ resonance show that the co-pol power is concentrated in the VV channel, again consistent with the frequency responses of Figure 2 , where the voltage magnitude is higher in the vertical channel than in the horizontal channel.

Although the ellipsoid and the cylinder have almost the same result for the 1 st resonance, the coincidence departs slightly for higher-order harmonics as the cylinder flat end geometry predominates around $1.3 \mathrm{GHz}$. This can be explained by the fact that the smaller detail of targets such as the flat ends start to be revealed at higher frequencies. 


\section{International Journal of Science and Research (IJSR) \\ ISSN (Online): 2319-7064}

Index Copernicus Value (2013): 6.14 | Impact Factor (2015): 6.391

The results for the rotated ellipsoid also show that the ellipsoid characteristic polarization states have undergone a linear orthogonal transformation of $45^{\circ}$ (i.e., $g_{1}$ and $g_{2}$ interchange values) which coincides with the physical rotation.

\subsection{Feature set}

Figure 3 shows a sample of distance measures for normal incidence. The feature set dimensions or variables will be the three distance measures $\left(\mathrm{d}_{1,2}, \mathrm{~d}_{1,3}, \mathrm{~d}_{2,3}\right)$ in this case, thus the feature set space is a three dimensional for the first three resonances. This can be beneficial in the classification stage, since the classification space dimensionality will always be less than the feature set dimensionality by one [33]. The feature space distributions of both targets are demonstrated in Figure 3 , where the feature set observations are plotted as function of azimuth variation from $1^{\circ}$ to $82^{\circ}$ with uniform sampling steps of size equal to $9^{\circ}$. The feature set distribution for both targets in Figure 3 clearly shows differences especially in the co-pol null and x-pol maximum portions of the feature set. One can observe some clustering especially near the origin, where the azimuth angle is near zero and the target geometry is viewed from the side along the $\mathrm{x}$-axis where both targets appear as a sphere. From Table I and Table II it is possible to conclude that: firstly, the ellipsoid metric distances are invariant to orientation along the radar direction; secondly, the cylinder distances departed of the ellipsoid distances; thirdly, in general, these distances can discriminate better between the targets than in the case for original (uniform) CPS.

\section{Conclusions}

In radar target recognition, the target should be represented by a robust (compact, immune to noise, etc..) feature set that enables a recognition operation to be simple and in the same time have sufficient accuracy. Therefore, a feature set based on CPS and their associated powers at the target natural resonant frequencies was developed for this purpose. Theoretically, a pre-selected modified CPS model was demonstrated for target recognition where these target s $^{\circ}$ CPS are modified by their respective power levels and distances between these respective CPS are used to develop a radar target feature set invariant to target orientation along antenna line-of-sight. This was demonstrated for two similar rigid targets where a resonance only feature set would experience difficulty separating the two targets. Apart from the capability of identifying similar targets, the procedure also provides physical interpretations that are sometimes lacking in other procedures. This work is believed to be the first step in a new approach to target feature selection that combines a polarimetry viewpoint with that of a target resonance description. Future work will concentrate designing a probabilistic model to evaluate the classification accuracy based on this feature set. The algorithm also needs to be evaluated for the presence of both clutter and noise, and subsequently for more complex targets in a measurement environment. Another important issue to be addressed in future work is to reduce the radar feature set dependency on target-radar aspect angle.

Table I: Modified characteristic polarization states for the geometry specified in the first column. The optimal polarization states are weighted according to their power levels.

\begin{tabular}{|c|c|c|c|c|}
\hline Target & Weighted CPS & $1^{\text {st }}$ resonance & $2^{\text {nd }}$ resonance & $3^{\text {rd }}$ resonance \\
\hline Ellipsoid & $\mathrm{g}^{\mathrm{mo}}\left(\mathrm{cm}_{1}\right)$ & $(1,0,0)$ & $(1,0,0)$ & $(1,0,0)$ \\
& $\mathrm{g}^{\mathrm{mo}}\left(\mathrm{cm}_{2}\right)$ & $(-0.04,0,0)$ & $(-0.82,0,0)$ & $(-0.47,0,0)$ \\
& $\mathrm{g}^{\mathrm{mo}}\left(\mathrm{cm}_{1}\right)$ & $(-0.14,-0.07,-0.144$ & $(-0.04,-0.38,-0.83$ & $(-0.13,-0.12,-0.67$ \\
& $\mathrm{g}^{\mathrm{mo}}\left(\mathrm{xm}_{1}\right)$ & ) & ) & ) \\
& & $(0,-0.22,-0.472)$ & $(0,-0.38,-0.83)$ & $(0,-0.13,-0.73)$ \\
\hline \multirow{2}{*}{ Ellipsoid with 45 } & & $(0,-1,0)$ & $(0,-1,0)$ & $(0,-1,0)$ \\
orientation & $\mathrm{g}^{\mathrm{mo}}\left(\mathrm{cm}_{1}\right)$ & $(0,0.04,0)$ & $(0,0.82,0)$ & $(0,0.47,0)$ \\
& $\mathrm{g}^{\mathrm{mo}}\left(\mathrm{cm}_{2}\right)$ & $(-0.07,0.14,-0.144)$ & $(-0.38,0.04,-0.83)$ & $(-0.12,0.13,-0.67)$ \\
& $\mathrm{g}^{\mathrm{mo}}\left(\mathrm{cn}_{1}\right)$ & $(-0.22,0,-0.472)$ & $(-0.38,0,-0.83)$ & $(-0.13,0,-0.73)$ \\
\hline Cylinder & $\mathrm{g}^{\mathrm{mo}}\left(\mathrm{xm}_{1}\right)$ & $(1,0,0)$ & $(0.56,0,0)$ & $(1,0,0)$ \\
& $\mathrm{g}^{\mathrm{mo}}\left(\mathrm{cm}_{1}\right)$ & $(-0.07,0,0)$ & $(-1,0,0)$ & $(-0.6,0,0)$ \\
& $\mathrm{g}^{\mathrm{mo}}\left(\mathrm{cm}_{2}\right)$ & $(-0.15,-0.09,-0.2)$ & $(0.1,-0.3,-0.71)$ & $(-0.1,0,-0.77)$ \\
& $\mathrm{g}^{\mathrm{mo}}\left(\mathrm{cm}_{1}\right)$ & $(0,-0.23,-0.49)$ & $(0,-0.3,-0.74)$ & $(0,0,-0.8)$ \\
\hline
\end{tabular}

Table II: Scaled Distance Measures of Ellipsoid and Cylinder.

\begin{tabular}{|c|c|c|c|}
\hline Modified CPS & $\begin{array}{c}45^{\circ} \text { Rotated Ellipsoid } \\
\mathrm{d}_{1,2}, \mathrm{~d}_{1,3}, \mathrm{~d}_{2,3}\end{array}$ & $\begin{array}{c}\text { Ellipsoid } \\
\mathrm{d}_{1,2}, \mathrm{~d}_{1,3}, \mathrm{~d}_{2,3}\end{array}$ & $\begin{array}{c}\text { Cylinder } \\
\mathrm{d}_{1,2}, \mathrm{~d}_{1,3}, \mathrm{~d}_{2,3}\end{array}$ \\
\hline $\mathrm{g}^{\mathrm{mo}}\left(\mathrm{cm}_{1}\right)$ & $(0,0,0)$ & $(0,0,0)$ & $(0.4,0,0.4)$ \\
\hline $\mathrm{g}^{\mathrm{mo}}\left(\mathrm{cm}_{2}\right)$ & $(0.78,0.43,0.35)$ & $(0.78,0.43,0.35)$ & $(0.93,0.53,0.4)$ \\
\hline $\mathrm{g}^{\mathrm{mo}}\left(\mathrm{cn}_{1}\right)$ & $(0.75,0.52,0.31)$ & $(0.75,0.52,0.31)$ & $(0.6,0.58,0.36)$ \\
\hline $\mathrm{g}^{\mathrm{mo}}\left(\mathrm{xm}_{1}\right)$ & $(0.39,0.27,0.27)$ & $(0.39,0.27,0.27)$ & $(0.26,0.4,0.32)$ \\
\hline
\end{tabular}



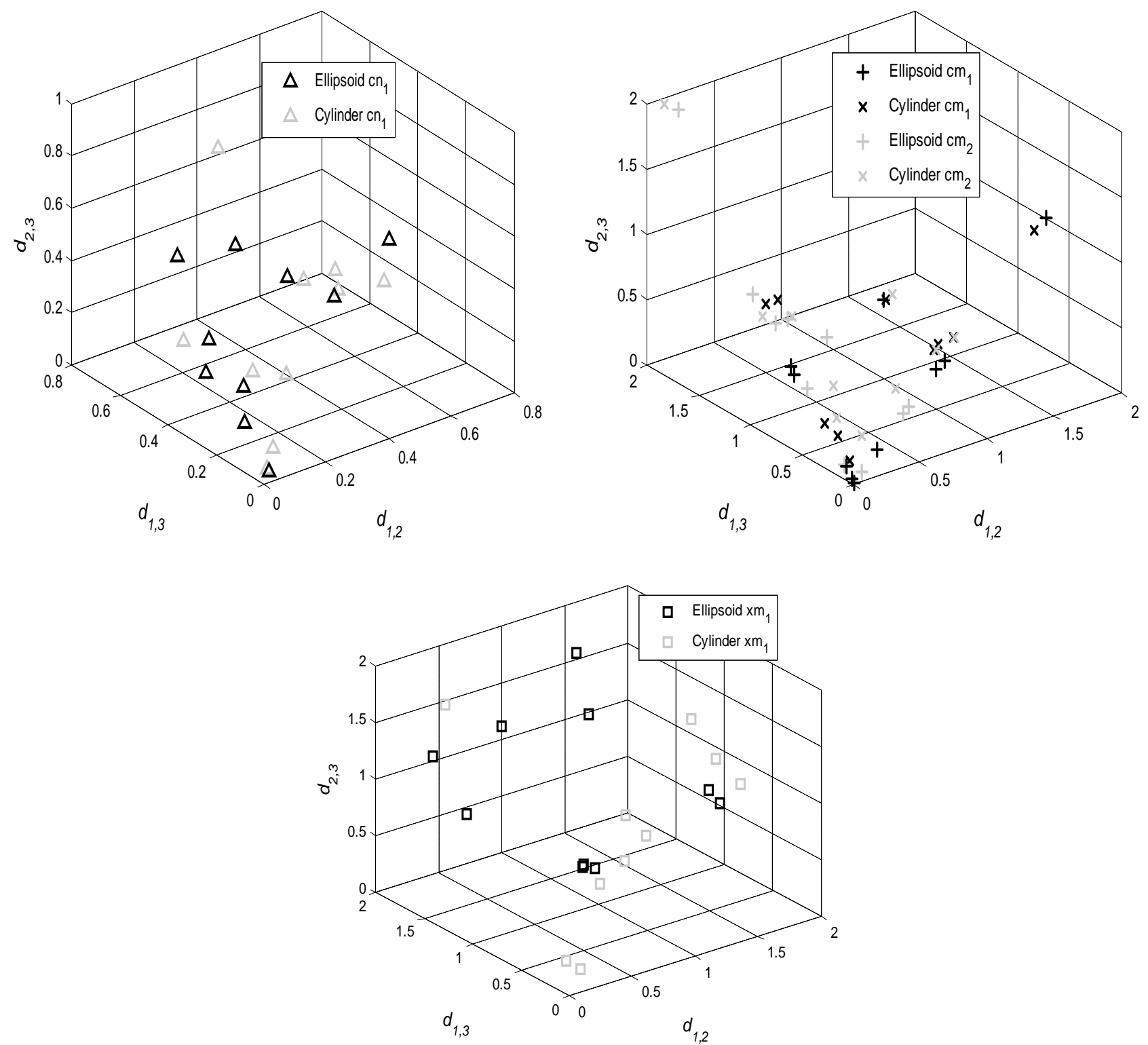

Figure 3: The modified CPS distance distributions in a 3D Euclidean space as a function of azimuth angle variation (1 to 82 with $9^{\circ}$ sampling steps), for Ellipsoid (black), Cylinder (gray)

\section{Acknowledgment}

This work was supported and funded by The Public Authority of Education and Training, research project No (TS-11-14).

\section{References}

[1] G. Galati, Advanced radar techniques and systems London : Peter Peregrinus on behalf of the Institution of Electrical Engineers, c1993., 1993.

[2] C. E. Baum, "Signature-based target identification and pattern recognition," Antennas and Propagation Magazine, IEEE, vol. 36, pp. 44-51, 1994.

[3] J. D. Morales, D. Blanco, D. P. Ruiz, and M. C. Carrion, "Radar-Target Identification via Exponential Extinction-Pulse Synthesis," Antennas and Propagation, IEEE Transactions on, vol. 55, pp. 2064-2072, 2007.
[4] H. S. Lui and N. V. Z. Shuley, "Radar Target Identification Using a "Banded" E-pulse Technique," Antennas and Propagation, IEEE Transactions on, vol. 54, pp. 3874-3881, 2006.

[5] J. E. Mooney, Z. Ding, and L. S. Riggs, "Performance analysis of an automated E-pulse target discrimination scheme," Antennas and Propagation, IEEE Transactions on, vol. 48, pp. 619-628, 2000.

[6] T. K. Sarkar and O. Pereira, "Using the matrix pencil method to estimate the parameters of a sum of complex exponentials," Antennas and Propagation Magazine, IEEE, vol. 37, pp. 48-55, 1995.

\section{Volume 5 Issue 4, April 2016}


[7] F. Aldhubaib, "Polarization Angles As A Radar Feature Set " International Journal of Enhanced Research in Science Technology \& Engineering (IJERSTE), vol. 5, April - 20162016.

[8] F. F. H. Aldhubaib and N. V. Z. Shuley, "Characteristic Polarization States Estimation in an Ultrawideband Context: A Frequency Approach," IEEE Transactions on Geoscience and Remote Sensing, vol. 47, pp. 2808-2817, 2009.

[9] W.-L. Yan and W. Boerner, "Optimal Polarization States Determination of the Stokes Reflection Matrices [Mp] for the Cohehernt case, and of the Muller Matrix [M] for the Partially Polarized Case," Journal of Electromagnetism Waves and Applications, vol. 5, pp. 1123-1149, 1991.

[10]D. Giuli, "Polarization diversity in radars," Proceedings of the IEEE, vol. 74, pp. 245-269, 1986.

[11] H. Mott, Remote sensing with polarimetric radar New York, N.Y: Wiley-IEEE; Chichester : John Wiley 2007.

\section{Author Profile}

Faisal F. H. Aldhubaib received his B.E. degree in electrical and computer engineering and then M.Sc. degree in radio and microwave engineering from the University of Leeds, UK, in 1995 and 1997, respectively. Then in 2010, he received his $\mathrm{PhD}$ in Electromagnetic and Imaging research at the School of Information Technology and Electrical Engineering (ITEE) from the University of Queensland, Australia. Currently, he is an assistant professor working in the college of technological studies, PAAET, Kuwait. 